\title{
INEQUALITIES INVOLVING MULTIVARIATE CONVEX FUNCTIONS II
}

\author{
EDWARD NEUMAN
}

(Communicated by R. Daniel Mauldin)

\begin{abstract}
In this paper we offer some inequalities involving multivariate convex functions. Among other things a refinement of classical Jensen's inequality as well as an extension of Fejér's inequality to the case of $s$-variate $(s \geq 1)$ functions are included. These results are obtained with the aid of the generalized simplex splines.
\end{abstract}

\section{INTRODUCTION}

The history of convex functions is long and laden with detail. These play an important role in mathematical analysis, applied mathematics, probability theory, and even in geometry. Among many known results about convex functions the following one is fundamental. Let $f$ be an $s$-variate continuous convex function on an open subset $U$ of $\mathbb{R}^{s}(s \geq 1)$. Then

$$
f\left(\sum_{m=0}^{n} \omega_{m} x^{m}\right) \leq \sum_{m=0}^{n} \omega_{m} f\left(x^{m}\right),
$$

where $\omega_{m}>0$, for all $m ; \omega_{0}+\cdots+\omega_{n}=1 ; x^{0}, \ldots, x^{n} \in U$. This classical result is essentially due to Jensen (see, e.g., [11]). A list of applications of this inequality is almost endless.

In this paper, which is a continuation of our earlier work [10], we give some inequalities for multivariate convex functions. The main results are obtained with the aid of the generalized simplex splines. In $\S 2$ we introduce notation and definitions that will be used in the sequel. Some auxiliary results are contained in $\S 3$. In $\S 4$, among other things, we give a refinement of Jensen's inequality and in addition, we offer an extension of Fejér's inequality (see $[6,9])$ to the case of $s$-variate functions.

Received by the editors September 26, 1988.

1980 Mathematics Subject Classification (1985 Revision). Primary 26B25, 26D15; Secondary $41 \mathrm{~A} 15,33 \mathrm{~A} 35$.

Key words and phrases. Multivariate convex functions, density functions, generalized simplex splines, $R$-hypergeometric functions. 


\section{NOTATION AND DEFINITIONS}

Let us introduce some notation and definitions which will be used throughout the sequel. By $x, y, \ldots$, we denote elements of Euclidean space $\mathbf{R}^{s}(s \geq 1)$, i.e., $x=\left(x_{1}, \ldots, x_{s}\right)$. Superscripts are used to number vectors. The inner product of $x, y \in \mathbf{R}^{s}$ is denoted by $x \cdot y=\sum_{l=1}^{s} x_{l} y_{l}$. For a given set $X \subset \mathbf{R}^{s}$ the symbols $\operatorname{vol}_{s}(X)$ and $[X]$ mean the $s$-dimensional Lebesgue measure and the convex hull of $X$, respectively. We use standard multi-index notation, i.e., for $\alpha \in \mathbf{Z}_{+}^{s},|\alpha|=\alpha_{1}+\cdots+\alpha_{s}, \alpha !=\alpha_{1} ! \cdots \alpha_{s} !, x^{\alpha}=x_{1}^{\alpha_{1}} \cdots x_{s}^{\alpha_{s}}$. Thus

$$
P_{m}\left(\mathbf{R}^{s}\right)=\left\{\sum_{|\alpha| \leq m} c_{\alpha} x^{\alpha}: c_{\alpha} \in \mathbf{R}\right\}
$$

is the space of all polynomials of (total) degree $\leq m$. By

$$
S^{n}=\left\{t=\left(t_{1}, \ldots, t_{n}\right) \in \mathbf{R}^{n}: t_{j} \geq 0, \text { for all } j, \sum_{j=1}^{n} t_{j} \leq 1\right\}
$$

we denote the standard $n$-simplex.

Let $\varphi$ be a nonnegative function on $\mathbf{R}^{n}$ with $\operatorname{supp} \varphi=S^{n}$. We assume $\varphi(t)$ is continuous for any $t \in \operatorname{Int} S^{n}$ and also that

$$
\int_{S^{n}} \varphi(t) d t=1 \quad\left(d t=d t_{1} \cdots d t_{n}\right) .
$$

Thus $\varphi$ is a density (or weight) function on $S^{n}$.

For $X=\left\{x^{0}, \ldots, x^{n}\right\} \subset \mathbf{R}^{s} \quad(n \geq s)$ with $\operatorname{vol}_{s}([X]) \neq 0$, the generalized simplex spline $M_{\varphi}(\cdot \mid X)$ is defined by requiring that

$$
\int_{\mathbf{R}^{s}} f(x) M_{\varphi}(x \mid X) d x=\int_{S^{n}} f(X t) \varphi(t) d t
$$

for all $f \in C\left(\mathbf{R}^{s}\right)$ (see [4]). Here $d x=d x_{1} \cdots d x_{s}, X t:=x^{0}+t_{1}\left(x^{1}-x^{0}\right)+\cdots+$ $t_{n}\left(x^{n}-x^{0}\right)$. It follows from [4, p. 34], that $M_{\varphi}(\cdot \mid X)$ is a multivariate density function on $\mathbf{R}^{s}$ with $\operatorname{supp} M_{\varphi}(\cdot \mid X)=[X]$. When $\varphi(t)=n ! \quad\left(t \in S^{n}\right)$ we will write $M(\cdot \mid X)$ instead of $M_{\varphi}(\cdot \mid X)$. The latter splines, commonly referred to as the simplex splines, have been introduced by de Boor in [1] and studied extensively by several researchers (see [4] and the references therein). A more general class of splines can be obtained choosing

$$
\begin{aligned}
\varphi(t) \equiv \varphi_{D}(t)=\frac{\Gamma\left(\alpha_{0}+\cdots+\alpha_{n}\right)}{\Gamma\left(\alpha_{0}\right) \cdots \Gamma\left(\alpha_{n}\right)} \prod_{j=0}^{n} t_{j}^{\alpha_{j}-1} \\
\quad\left(\alpha_{0}, \ldots, \alpha_{n}>0 ; t_{0}=1-t_{1}-\cdots-t_{n}\right) .
\end{aligned}
$$

In this case we will write $M_{D}(\cdot \mid X)$ for the corresponding spline function. Dahmen and Micchelli [5] called $M_{D}(\cdot \mid X)$ the generalized Dirichlet density. For $\alpha_{0}=\cdots=\alpha_{n}=1, M_{D}(\cdot \mid X)$ becomes $M(\cdot \mid X)$. 


\section{Lemmas}

For our further purposes we need the following.

Lemma 3.1. Let $X=\left\{x^{0}, \ldots, x^{s}\right\} \subset \mathbf{R}^{s}(s \geq 1)$ be such that $\operatorname{vol}_{s}([X]) \neq 0$. Further, let $\varphi$ be a density function on $S^{S}$. Then

$$
M_{\varphi}(x \mid X)=\left[s ! \operatorname{abs}\left(\operatorname{vol}_{s}([X])\right)\right]^{-1} \varphi\left(A_{1}^{-1} \cdot\left(x-x^{0}\right), \ldots, A_{s}^{-1} \cdot\left(x-x^{0}\right)\right)
$$

for any $x \in \operatorname{Int}[X]$. Here $A_{j}^{-1}(1 \leq j \leq s)$ stands for the $j$ th row of $A^{-1}$, where $A=\left(x^{1}-x^{0}, \ldots, x^{s}-x^{0}\right) \in \mathbf{R}^{s \times s}\left(x^{1}-x^{0}, \ldots, x^{s}-x^{0}\right.$-the columns of $A)$.

Proof. It is well known that

$$
\operatorname{det} A=s ! \operatorname{vol}_{s}([X]) \text {. }
$$

Since $\operatorname{vol}_{s}([X]) \neq 0$, the matrix $A$ is invertible. Taking into account that $\operatorname{supp} M_{\varphi}(\cdot \mid X)=[X]$, we can rewrite equation (2.1) as

$$
\int_{[X]} f(x) M_{\varphi}(x \mid X) d x=\int_{S^{s}} f\left(x^{0}+A t\right) \varphi(t) d t .
$$

Substituting $x=x^{0}+A t$ into the right-hand side of the last equation, taking into account that the Jacobian of this transformation equals $\operatorname{det} A^{-1}$, and next making use of (3.2), we obtain the assertion.

For a given subset $X=\left\{x^{0}, \ldots, x^{n}\right\}$ of $\mathbf{R}^{s}$ and for an arbitrary density $\varphi$ on $S^{n}$ we define

$$
m_{\varphi}(j, r, X)=\int_{S^{n}}\left(\sum_{m=0}^{n} t_{m} x_{j}^{m}\right)^{r} \varphi(t) d t .
$$

where $r \in \mathbf{R} ; 1 \leq j \leq s$. Here $x_{j}^{m}$ stands for the $j$ th component of the vector $x^{m}$. In the particular case, when $\varphi$ is the Dirichlet density (see (2.2)) and $r \neq 0$, the right-hand side of (3.3) becomes an $R$-hypergeometric function in the variables $x_{j}^{0}, \ldots, x_{j}^{n}$ (see [2]). The latter will be denoted by $m_{D}(j, r, X)$. For more details concerning these functions consult, e.g., [3].

In the case when $\operatorname{vol}_{s}([X]) \neq 0$ we can utilize $(2.1)$ to obtain

$$
m_{\varphi}(j, r, X)=\int_{\mathbf{R}^{s}}\left(x_{j}\right)^{r} M_{\varphi}(x \mid X) d x,
$$

where $x_{j}$ stands for the $j$ th component of $x$.

For our purposes we let

$$
\mu_{\varphi}(\beta)=\int_{S^{n}} \prod_{j=0}^{n} t_{j}^{\beta_{j}} \varphi\left(t_{1}, \ldots, t_{n}\right) d t_{1} \cdots d t_{n},
$$

where $\beta=\left(\beta_{0}, \ldots, \beta_{n}\right) \in \mathbf{Z}_{+}^{n+1} ;\left(t_{1}, \ldots, t_{n}\right) \in S^{n} ; t_{0}=1-t_{1}-\cdots-t_{n}$. We also let $z_{j}=\left(x_{j}^{0}, \ldots, x_{j}^{n}\right) \quad(1 \leq j \leq s)$ and

$$
\left(\begin{array}{l}
r \\
\beta
\end{array}\right)=\frac{r !}{\beta !} \quad \text { (the multinomial coefficient), }
$$

where $r \in \mathbf{Z}_{+},|\beta|=r$. 
The following result is essentially due to Carlson [2] who proved (3.7) and (3.8).

Lemma 3.2. Let $r \in \mathbf{Z}_{+}$and let $j=1,2, \ldots, s$. Then

$$
m_{\varphi}(j, r, X)=\sum_{|\beta|=r}\left(\begin{array}{l}
r \\
\beta
\end{array}\right) \mu_{\varphi}(\beta) z_{j}^{\beta},
$$

where the summation is extended over all multi-indices $\beta \in \mathbf{Z}_{+}^{n+1}$. If $\varphi=\varphi_{D}$, then

$$
m_{D}(j, r, X)=\frac{\Gamma(c) r !}{\Gamma(c+r)} \sum_{|\beta|=r} z_{j}^{\beta} \prod_{m=0}^{n} \frac{\Gamma\left(\alpha_{m}+\beta_{m}\right)}{\Gamma\left(\alpha_{m}\right) \beta_{m} !},
$$

with $c=\alpha_{0}+\cdots+\alpha_{n}\left(\alpha_{j}>0\right.$, for all $\left.j\right)$. If in addition $x^{m}>0$, for all $m$, then

$$
m_{D}(j,-c, X)=\prod_{m=0}^{n}\left(x_{j}^{m}\right)^{-\alpha_{m}}
$$

Since the proof of (3.6) is exactly the same as the proof of $[2,(3.1)]$, we omit further details.

We also need the following identity:

$$
\int_{\mathbf{R}^{s}} x_{j} M_{\varphi}(x \mid X) d x=\sum_{m=0}^{n} \omega_{m} x_{j}^{m} \quad(1 \leq j \leq s)
$$

which follows immediately from (3.4), (3.6), and (3.5) by letting $r=1$. Here

$$
\omega_{m}=\int_{S^{n}} t_{m} \varphi(t) d t \quad m=0,1, \ldots, n .
$$

It is clear that $\omega_{m}>0$, for all $m$, and also $\omega_{0}+\cdots+\omega_{n}=1$. In particular, when $\varphi=\varphi_{D}$,

$$
\omega_{m}=\alpha_{m} / c
$$

for all $m($ see [12]).

\section{Main Results}

In this section we offer some inequalities involving multivariate convex functions. Theorems 4.1 and 4.2 give generalizations of [10, Theorems 3.1 and $3.2]$.

We are ready now to state and prove the following.

Theorem 4.1. Let $f$ be a continuous convex function on $\mathbf{R}^{s}$. Further, let $\varphi$ be a density function on $S^{n}$ and let $X=\left\{x^{0}, \ldots, x^{n}\right\} \subset \mathbf{R}^{s} \quad(n \geq s \geq 1)$ with $\operatorname{vol}_{s}([X]) \neq 0$. Then for any $r_{1}, \ldots, r_{s} \in \mathbf{R} \backslash\{0\}$

$$
f\left[m_{\varphi}\left(1, r_{1}, X\right), \ldots, m_{\varphi}\left(s, r_{s}, X\right)\right] \leq \int_{\mathbf{R}^{s}} f\left[\left(x_{1}\right)^{r_{1}}, \ldots,\left(x_{s}\right)^{r_{s}}\right] M_{\varphi}(x \mid X) d x
$$

with equality if and only if $f \in P_{1}\left(\mathbf{R}^{s}\right)$. 
Proof. In order to establish (4.1) we utilize the following version of Jensen's inequality for multivariate convex functions:

$$
f\left[\int_{\mathbf{R}^{s}} g_{1} d \nu, \ldots, \int_{\mathbf{R}^{s}} g_{s} d \nu\right] \leq \int_{\mathbf{R}^{s}} f\left(g_{1}, \ldots, g_{s}\right) d \nu,
$$

where $g_{1}, \ldots, g_{s} \in C\left(\mathbf{R}^{s}\right)$ and $d \nu$ is a probability measure on $\mathbf{R}^{s}$ (see [8]). The equality holds in (4.2) if and only if $f \in P_{1}\left(\mathbf{R}^{s}\right)$. Now the assertion follows immediately from (4.2) and (3.4) by letting $g_{j}(x)=\left(x_{j}\right)^{r_{j}} \quad(1 \leq j \leq s)$ and $d \nu(x)=M_{\varphi}(x \mid X) d x$.

Theorem 4.2. Under the assumptions of Theorem 4.1,

$$
f\left(\sum_{m=0}^{n} \omega_{m} x^{m}\right) \leq \int_{\mathbf{R}^{s}} f(x) M_{\varphi}(x \mid X) d x \leq \sum_{m=0}^{n} \omega_{m} f\left(x^{m}\right),
$$

where the $\omega$ 's are given by (3.10). The inequalities in (4.3) become equalities if and only if $f \in P_{1}\left(\mathbf{R}^{s}\right)$.

Proof. For the proof of the left-hand side inequality of (4.3) we utilize Theorem 4.1 with $r_{1}=\cdots=r_{s}=1$. Hence, by virtue of (3.4) and (3.9),

$$
f\left(\sum_{m=0}^{n} \omega_{m} x^{m}\right)=f\left(\sum_{m=0}^{n} \omega_{m} x_{1}^{m}, \ldots, \sum_{m=0}^{n} \omega_{m} x_{s}^{m}\right) \leq \int_{\mathbf{R}^{s}} f(x) M_{\varphi}(x \mid X) d x .
$$

In order to show that the right-hand side inequality of (4.3) is valid we employ (2.1) and (3.10). This yields

$$
\begin{aligned}
\int_{\mathbf{R}^{s}} f(x) M_{\varphi}(x \mid X) d x & =\int_{S^{n}} f\left(\sum_{m=0}^{n} t_{m} x^{m}\right) \varphi(t) d t \\
& \leq \sum_{m=0}^{n} f\left(x^{m}\right) \int_{S^{n}} t_{m} \varphi(t) d t=\sum_{m=0}^{n} \omega_{m} f\left(x^{m}\right) .
\end{aligned}
$$

The last assertion of the theorem is obvious. The proof is completed.

In 1906 Fejér [6] proved that for any functions $f$ and $h$, both continuous in $(a, b)$, the inequality

$$
f\left(\frac{a+b}{2}\right) \leq \int_{a}^{b} f(x) h(x) d x / \int_{a}^{b} h(x) d x \leq \frac{1}{2}[f(a)+f(b)]
$$

holds provided, however, that $f$ is convex in $(a, b)$, that $h$ is positive and symmetric with respect to the straight line which contains the point $\left(\frac{1}{2}(a+b)\right.$, 0 ), and is perpendicular to the $x$-axis (see also [9]).

We will show below that Fejér's result can be extended to the case of multivariate functions.

Corollary 4.3 (Fejér's Inequality for Multivariate Functions). Let $X=\left\{x^{0}, \ldots\right.$, $\left.x^{s}\right\}$ be a subset of $\mathbf{R}^{s}(s \geq 1)$ with $\operatorname{vol}_{s}(\sigma) \neq 0$, where $\sigma=[X]$. Suppose that 
$f$ and $h$ are s-variate continuous functions on Int $\sigma$. If $f$ is convex on Int $\sigma$ and $h$ is positive on Int $\sigma$, then

$$
f\left(\sum_{m=0}^{s} \omega_{m} x^{m}\right) \leq \int_{\sigma} f(x) h(x) d x / \int_{\sigma} h(x) d x \leq \sum_{m=0}^{s} \omega_{m} f\left(x^{m}\right),
$$

where

$$
\omega_{m}=\int_{\sigma}\left[A_{m}^{-1} \cdot\left(x-x^{0}\right)\right] h(x) d x / \int_{\sigma} h(x) d x \quad(1 \leq m \leq s)
$$

and $\omega_{0}=1-\omega_{1}-\cdots-\omega_{s}$. Here $A_{m}^{-1}$ has the same meaning as in Lemma 3.1 .

Proof. We prove (4.4) and (4.5) assuming without loss of generality that the function $h$ is normalized to have the unit integral. It follows from Lemma 3.1 that for any density function on $S^{s}$ there is a unique generalized simplex spline $M_{\varphi}(\cdot \mid X)$ provided that $\operatorname{vol}_{s}(\sigma) \neq 0$. In order to establish the assertion (4.4) it is sufficient to apply Theorem 4.2 with $n=s$ and $M_{\varphi}(\cdot \mid X)=h(\cdot)$. For the proof of (4.5), with $h$ as assumed above, we employ (3.10), i.e.,

$$
\omega_{m}=\int_{S^{s}} t_{m} \varphi\left(t_{1}, \ldots, t_{s}\right) d t_{1} \cdots d t_{s} \quad(0 \leq m \leq s) .
$$

For a given $t=\left(t_{1}, \ldots, t_{s}\right) \in S^{s}$ we introduce a vector $x=\left(x_{1}, \ldots, x_{s}\right)$, where $t=A^{-1}\left(x-x^{0}\right)$ with $A$ as defined in Lemma 3.1. It is clear that $x \in \sigma$. Taking into account that the Jacobian of this transformation equals $\operatorname{det} A^{-1}$ and next making use of (4.6) and (3.2), we obtain

$$
\begin{aligned}
\omega_{m}= & \int_{\sigma}\left[A_{m}^{-1} \cdot\left(x-x^{0}\right)\right]\left[s ! \operatorname{abs}\left(\operatorname{vol}_{s}(\sigma)\right)\right]^{-1} \\
& \times \varphi\left(A_{1}^{-1} \cdot\left(x-x^{0}\right), \ldots, A_{s}^{-1} \cdot\left(x-x^{0}\right)\right) d x \\
= & \int_{\sigma}\left[A_{m}^{-1} \cdot\left(x-x^{0}\right)\right] M_{\varphi}(x \mid X) d x=\int_{\sigma}\left[A_{m}^{-1} \cdot\left(x-x^{0}\right)\right] h(x) d x
\end{aligned}
$$

which completes the proof.

We will show now that Fejér's inequality can be easily derived from Corollary 4.3. To this aim it is sufficient to insert $s=1, x^{0}=a, x^{1}=b \quad(a \neq b)$ into (4.4)-(4.5). We have $A_{1}^{-1} \cdot\left(x-x^{0}\right)=(x-a) /(b-a)$. Hence (4.5) yields

$$
\omega_{1}=\int_{a}^{b} \frac{x-a}{b-a} h(x) d x / \int_{a}^{b} h(x) d x .
$$

Substituting $x:=a+b-x$ and taking into account that $h$ is assumed to be an even function with respect to the midpoint $(a+b) / 2$, we obtain

$$
\omega_{1}=\int_{a}^{b} \frac{b-x}{b-a} h(x) d x / \int_{a}^{b} h(x) d x=\omega_{0} .
$$

Since $\omega_{0}+\omega_{1}=1, \omega_{0}=\omega_{1}=\frac{1}{2}$. Hence the desired result follows. 
Corollary 4.3 can be also utilized when deriving Hermite's inequality for multivariate convex functions:

$$
f\left(\frac{1}{s+1} \sum_{m=0}^{s} x^{m}\right) \leq \frac{1}{\operatorname{abs}\left(\operatorname{vol}_{s}(\sigma)\right)} \int_{\sigma} f(x) d x \leq \frac{1}{s+1} \sum_{m=0}^{s} f\left(x^{m}\right)
$$

(see [10]). To this aim we insert $h(x)=1$ into (4.4) and (4.5). It follows from (4.5) that

$$
\omega_{m}=\frac{1}{\operatorname{abs}\left(\operatorname{vol}_{s}(\sigma)\right)} \int_{\sigma}\left[A_{m}^{-1} \cdot\left(x-x^{0}\right)\right] d x=s ! \int_{S^{s}} t_{m} d t_{1} \cdots d t_{s}
$$

for $m=1,2, \ldots, s$. Since

$$
\int_{S^{s}} t_{m} d t_{1} \cdots d t_{s}=1 /(s+1) !
$$

(see, e.g., [12]), $\omega_{1}=\cdots=\omega_{s}=1 /(s+1)$. Hence $\omega_{0}=1 /(s+1)$, which proves (4.7).

Before the next theorem we introduce more notation. To this end we will assume that $\varphi=\varphi_{D}$-the Dirichlet density on $S^{n}$ with parameters $\alpha_{0}, \ldots, \alpha_{n} \geq$ 1 (see Equation (2.2)). By $\varphi_{D_{j}}$ we denote Dirichlet's density on $S^{n-1}$ with parameters $\alpha_{0}, \ldots, \alpha_{j-1}, \alpha_{j+1}, \ldots, \alpha_{n}(0 \leq j \leq n ; n>s \geq 1)$. The symbol $\varphi_{\bar{D}_{j}}$ stands for the Dirichlet density on $S^{n}$ with parameters $\alpha_{0}, \ldots, \alpha_{j-1}, \alpha_{j}-$ $1, \alpha_{j+1}, \ldots, \alpha_{n}$. The corresponding generalized simplex splines are denoted by $M_{D_{j}}\left(\cdot \mid X_{j}\right)$ and $M_{\bar{D}_{j}}(\cdot \mid X)$, respectively. Here $X_{j}=\left\{x^{0}, \ldots, x^{j-1}, x^{j+1}, \ldots\right.$, $\left.x^{n}\right\} \subset \mathbf{R}^{s} \quad(0 \leq j \leq n ; n>s \geq 1)$. The following recurrence formula plays a crucial role in our further investigations:

$$
\begin{aligned}
\gamma_{j} \delta_{\left(\alpha_{j}-1\right)} M_{D_{j}}\left(\cdot \mid X_{j}\right)= & D_{x^{j}-x} M_{D}(\cdot \mid X)+(c-s-1) M_{D}(\cdot \mid X) \\
& +(c-1)\left(\delta_{\left(\alpha_{j}-1\right)}-1\right) M_{\bar{D}_{j}}(\cdot \mid X)
\end{aligned}
$$

(see [7]). This formula holds true provided $\operatorname{vol}_{s}\left(\left[X_{j}\right]\right) \neq 0$ for some $0 \leq j \leq n$. In (4.8),

$$
D_{u} g(x)=\sum_{l=1}^{s} u_{l} \frac{\partial g(x)}{\partial x_{l}}
$$

denotes the directional derivative of $g \in C^{1}\left(\mathbf{R}^{s}\right)$ in the direction $u \in \mathbf{R}^{s}$; $\gamma_{j}=\Gamma(c) / \Gamma\left(\alpha_{j}\right) \Gamma\left(c-\alpha_{j}\right):$

$$
\delta_{v}= \begin{cases}1 & v=0 \\ 0 & \text { otherwise }\end{cases}
$$

Let $\lambda_{0}, \ldots, \lambda_{n} \in \mathbf{R}$ with $\lambda_{0}+\cdots+\lambda_{n}=1$. Multiplying both sides of (4.8) by $\lambda_{j}$ and next summing over all $j$, we obtain, for any $X \subset \mathbf{R}^{s}$ with $\operatorname{vol}_{s}([X]) \neq 0$, 


$$
\begin{aligned}
\sum_{j=0}^{n} \lambda_{j} \gamma_{j} \delta_{\left(\alpha_{j}-1\right)} M_{D_{j}}\left(\cdot \mid X_{j}\right)= & D_{y-x} M_{D}(\cdot \mid X)+(c-s-1) M_{D}(\cdot \mid X) \\
& +(c-1) \sum_{j=0}^{n} \lambda_{j}\left(\delta_{\left(\alpha_{j}-1\right)}-1\right) M_{\bar{D}_{j}}(\cdot \mid X),
\end{aligned}
$$

where

$$
y=\sum_{j=0}^{n} \lambda_{j} x^{j} .
$$

The recursion (4.9) is valid for any $x \in[X]$ provided, however, that $\operatorname{vol}_{s}\left(\left[X_{j}\right]\right)$ $\neq 0$, for all $j$.

We are now in a position to state and prove the following:

Theorem 4.4. Let $X=\left\{x^{0}, \ldots, x^{n}\right\} \subset \mathbf{R}^{s} \quad(n>s \geq 1)$ with $\operatorname{vol}_{s}\left(\left[X_{j}\right]\right) \neq 0$, for all $j$. Further, let $\lambda_{0}, \ldots, \lambda_{n}$ be arbitrary real numbers with $\lambda_{0}+\cdots+\lambda_{n}=1$. If $f \in C^{1}\left(\mathbf{R}^{s}\right)$ is a convex function, then for $\alpha_{0}, \ldots, \alpha_{n} \geq 1$

$$
\begin{aligned}
(c-1) & \int_{\mathbf{R}^{s}} f(x)\left[M_{D}(x \mid X)+\sum_{j=0}^{n} \lambda_{j}\left(\delta_{\left(\alpha_{j}-1\right)}-1\right) M_{\bar{D}_{j}}(x \mid X)\right] d x \\
\leq & \sum_{j=0}^{n} \lambda_{j} \gamma_{j} \delta_{\left(\alpha_{j}-1\right)} \int_{\mathbf{R}^{s}} f(x) M_{D_{j}}\left(x \mid X_{j}\right) d x
\end{aligned}
$$

if and only if

$$
\sum_{j=0}^{n} \omega_{j} x^{j}=\sum_{j=0}^{n} \lambda_{j} x^{j}
$$

where the $\omega$ 's are given by (3.11). If this condition is satisfied, then equality holds in (4.11) if and only if $f \in P_{1}\left(\mathbf{R}^{s}\right)$.

Proof. We employ the recursion (4.9). Multiplying both sides by $f(x)$ and next taking integrals over $\mathbf{R}^{s}$, we obtain

$$
\sum_{j=0}^{n} \lambda_{j} \gamma_{j} \delta_{\left(\alpha_{j}-1\right)} \int_{\mathbf{R}^{s}} f(x) M_{D_{j}}\left(x \mid X_{j}\right) d x=I+J+K,
$$

where

$$
\begin{aligned}
& I=\int_{\mathbf{R}^{s}} f(x) D_{y-x} M_{D}(x \mid X) d x, \\
& J=(c-s-1) \int_{\mathbf{R}^{s}} f(x) M_{D}(x \mid X) d x,
\end{aligned}
$$

and

$$
K=(c-1) \sum_{j=0}^{n} \lambda_{j}\left(\delta_{\left(\alpha_{j}-1\right)}-1\right) \int_{\mathbf{R}^{s}} f(x) M_{\bar{D}_{j}}(x \mid X) d x .
$$


In order to establish (4.11) we will find a lower bound on I. Applying the product rule we obtain

$$
\begin{aligned}
I & =\sum_{l=1}^{s} \int_{\mathbf{R}^{s}} f(x)\left(y_{1}-x_{1}\right) \frac{\partial}{\partial x_{1}} M_{D}(x \mid X) d x \\
& =\sum_{l=1}^{s} \int_{\mathbf{R}^{s}}\left\{\operatorname{div} F(x)-M_{D}(x \mid X)\left[\left(y_{1}-x_{1}\right) \frac{\partial f(x)}{\partial x_{1}}-f(x)\right]\right\} d x,
\end{aligned}
$$

where $F(x)=\left\{f(x)\left(y_{1}-x_{1}\right) M_{D}(x \mid X), \ldots, f(x)\left(y_{s}-x_{s}\right) M_{D}(x \mid X)\right\}$ is the vector field. Since $M_{D}(\cdot \mid X)$ has a local support there exists a closed bounded subset $\Omega$ of $\mathbf{R}^{S}$ such that $\operatorname{supp} M_{D}(\cdot \mid X) \subseteq \Omega$ and $F(x)=0$ for $x \in \partial \dot{\Omega}$ (the boundary of $\Omega$ ). Application of the divergence theorem yields

$$
\int_{\mathbf{R}^{s}} \operatorname{div} F(x) d x=0 .
$$

Hence

$$
I=s \int_{\mathbf{R}^{s}} f(x) M_{D}(x \mid X) d x+\sum_{l=1}^{s} \int_{\mathbf{R}^{s}} M_{D}(x \mid X)\left(x_{1}-y_{1}\right) \frac{\partial f(x)}{\partial x_{1}} d x .
$$

To obtain a lower bound on $I$, we utilize the well-known inequality for the multivariate convex functions

$$
\sum_{l=1}^{s}\left(x_{l}-y_{l}\right) \frac{\partial f(x)}{\partial x_{l}} \geq \sum_{l=1}^{s}\left(x_{l}-y_{l}\right) \frac{\partial f(y)}{\partial x_{l}}
$$

where $x, y \in \mathbf{R}^{s}$. If $x \neq y$, then the inequality is strict if and only if $f \notin$ $P_{1}\left(\mathbf{R}^{s}\right)$. Thus

$$
I \geq s \int_{\mathbf{R}^{s}} f(x) M_{D}(x \mid X) d x+\sum_{l=1}^{s} \frac{\partial f(y)}{\partial x_{l}} \int_{\mathbf{R}^{s}}\left(x_{l}-y_{l}\right) M_{D}(x \mid X) d x
$$

for any $y \in \mathbf{R}^{s}$. Assume $y$ is given by (4.10). Then, by virtue of (3.9),

$$
\begin{aligned}
I & \geq s \int_{\mathbf{R}^{s}} f(x) M_{D}(x \mid X) d x+\sum_{l=1}^{s} \frac{\partial f(y)}{\partial x_{l}}\left[\sum_{j=0}^{n} \omega_{j} x_{l}^{j}-\sum_{j=0}^{n} \lambda_{j} x_{l}^{j}\right] \\
& =s \int_{\mathbf{R}^{s}} f(x) M_{D}(x \mid X) d x,
\end{aligned}
$$

where the last equality holds if and only if the condition (4.12) is satisfied. Combining this with (4.14) and (4.15) one obtains

$$
I+J+K \geq(c-1) \int_{\mathbf{R}^{s}} f(x)\left[M_{D}(x \mid X)+\sum_{j=0}^{n} \lambda_{j}\left(\delta_{\left(\alpha_{j}-1\right)}-1\right) M_{\bar{D}_{j}}(x \mid X)\right] d x .
$$

This in conjunction with (4.13) yields the inequality (4.11). The last assertion of the theorem is an immediate consequence of (4.16) and (4.17). This completes the proof. 


\section{ACKNOWLEDGMENT}

The author wishes to thank Professor Josip Pečaric' for a comment concerning the proof of Theorem 4.4.

\section{REFERENCES}

1. C. de Boor, Splines as linear combinations of B-splines: a survey, in Approximation Theory II (G. G. Lorentz, C. K. Chui, and L. L. Schumaker, eds.), Academic Press, New York, 1976, pp. 1-47.

2. B. C. Carlson, A hypergeometric mean value, Proc. Amer. Math. Soc. 16 (1965), 759-766.

3. __ Special functions of applied mathematics, Academic Press, New York, 1977.

4. W. Dahmen and C. A. Micchelli, Recent progress in multivariate splines, in Approximation Theory IV (C. K. Chui, L. L. Schumaker, and J. D. Ward, eds.), Academic Press, New York, 1983, pp. 27-121.

5. __ Statistical encounters with B-splines, Contemp. Math. 59 (1986), 17-48.

6. L. Fejér, Über die Fourierreihen II, Math. Naturwiss. Anz. Ungar. Akad. Wiss. 24 (1906), 369-390.

7. S. Karlin, C. A. Micchelli, and Y. Rinot, Multivariate splines: probabilistic perspective, J. Multiv. Anal. 20 (1986), 69-90.

8. E. J. McShane, Jensen's inequality, Bull. Amer. Math. Soc. 43 (1937), 521-527.

9. D. S. Mitrinovic' and I. B. Lackovic' ${ }^{\prime}$, Hermite and convexity, Aequationes Math. 28 (1985), 229-232.

10. E. Neuman and J. Pečaric', Inequalities involving multivariate convex functions, J. Math. Anal. Appl. 137 (1989), 541-549.

11. A. W. Roberts and D. E. Varberg, Convex functions, Academic Press, New York and London, 1973.

12. E. T. Whittaker and G. N. Watson, A course of modern analysis, 4th ed., Cambridge University Press, Cambridge, 1927.

Department of Mathematics, Southern Illinois University at Carbondale, CarbonDALE, ILLINOIS 62901-4408 